\title{
Wir Gesundheitsverkäufer
}

\author{
Hanswerner Iff \\ Dr. med., Facharzt für Innere Medizin und Pneumologie, Mitglied FMH
}

Als «Hommage» an das 20-jährige Bestehen unserer Standesordnung möchte ich mir einige Gedanken zu zwei für unser Tun äusserst wichtigen Begriffen machen. Es handelt sich um «krank» und "gesund», um «Krankheit» und «Gesundheit». Dabei knüpfe ich an Diskussionen an, die wir vor 20 Jahren in der Arbeitsgruppe FMH führten. Es ging um die beste Formulierung der Aufgaben des Arztes und der Ärztin. Dieser Artikel wurde schliesslich folgendermassen abgefasst: Es ist Aufgabe des Arztes und der Ärztin, menschliches Leben zu schützen, Gesundheit zu fördern und zu erhalten, Krankheiten zu behandeln, Leiden zu lindern und Sterbenden beizustehen. Wir fanden rasch zu den Tätigkeitswörtern zu «menschliches Leben», «Leiden» und «Sterben», hatten aber einige Mühen, die passenden Verben zu «Gesundheit» und «Krankheit» zu finden. «Heilen», «bekämpfen», «verhindern» oder «behandeln» wir Krankheiten, «stellen» wir Gesundheit "wieder her», «fördern» oder «erhalten» wir sie? So ist in einem Protokoll einer Sitzung der Arbeitsgruppe von 1996 auch zu lesen, dass «Gesundheitsförderung» bedeutungsgleich sei mit «Krankheitsverhinderung». Dieser Eintrag mag unsere damaligen Diskussionen

\section{Résumé}

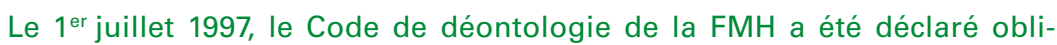
gatoire. II remplaçait les codes de déontologie des sociétés cantonales de médecine en vigueur jusque-là. Le Code de déontologie de la FMH fête donc ses 20 ans d'existence.

En tant que président du groupe de travail de l'époque, je ne suis pas bien placé pour juger à présent des réglementations clarificatrices qu'il a apportées à la FMH. Son effet a surtout été perceptible dans les évaluations des commissions de déontologie des sociétés cantonales de médecine et spécialisées. II ne m'appartient donc pas de proposer une critique de ce code d'un point de vue actuel. Je n'en pense pas moins qu'une telle analyse pourrait être tout à fait appropriée. Je suis par exemple stupéfait du nombre d'institutions et d'instances qui surveillent la pratique médicale et sont aujourd'hui sans lien avec le Code de déontologie de la FMH.

L'article "Nous, les marchands de santé» est né d'une brève discussion que nous avons eue il y a 20 ans au sein de notre groupe de travail, mais qui n'a désormais plus qu'un rapport marginal avec notre Code de déontologie. Il convient cependant comme hommage à ses 20 ans d'existence. illustrieren. Auch heute noch scheint mir ein Nachdenken über «krank» und «gesund» wichtig und nützlich. Dieses hat auch eine moralische Seite, die gut zu einem Rückblick auf das 20-jährige Jubiläum der Standesordnung FMH passt.

\section{Wandel im Sprachgebrauch}

Während den Jahrzehnten meiner ärztlichen Tätigkeit ist sicher nicht nur mir aufgefallen, dass ein überraschender Wandel im deutschen Sprachgebrauch zwischen «krank» und "gesund» stattgefunden hat. Während dieser Zeit wurde «krank» immer mehr durch "gesund" ersetzt. Es entgeht meiner Kenntnis, ob solche Bedeutungswechsel von Wörtern, die Gegensätzliches beinhalten, ein sprachwissenschaftlich bekanntes Phänomen sind. Derjenige von krank zu gesund ist, vor allem im deutschen Sprachbereich, irritierende Tatsache. So wurden in den vergangenen Jahrzehnten aus Krankenanstalten Gesundheitszentren, die Krankenschwester zuerst zur Gesundheitsschwester, dann zur Fachangestellten für Gesundheit, Krankengesetze zu Gesundheitsgesetzen, die hausärztliche Praxis zur «HMO» (gesundheitserhaltendes Unternehmen), der Krankenkassenverband zu santésuisse und Krankenkassen zu Gesundheitskassen. Krankenkosten sind nun Kosten für die Gesundheit, Big Data liefern heute unzählige Gesundheitsstatistiken, die aber Daten zu Krankheiten sind. Immerhin wird dieser frappante Wortwechsel, der praktisch ohne Bedeutungsänderungen stattfand, etwas relativiert, wenn die aus dem Lateinischen stammenden Krankheits- oder eben Gesundheitsbegriffe angeschaut werden. Ähnliche Veränderungen können im Französischen nicht gleichermassen nachgewiesen werden.

Die Entwicklung in der deutschen Sprache erstaunt. Mir gibt sie vor allem zu denken, weil sie ganz vergessen macht, dass Gesundheit vor allem ein Gut ist, das uns gegeben ist und das wir nicht erwerben müssen, und nur die Krankheit das Unerwünschte ist. Mit der Evolution haben auch wir Menschen in Millionen von Jahren eine gute Gesundheit erhalten. Durch die Jahre der Entwicklung von Leben auf dieser Erde hat sich auch unsere Gesundheit, also die Möglichkeit, gesund zu sein und zu bleiben, gebildet. Die Gesundheit ist 
damit bereits in allen Lebewesen und auch in uns enthalten. Wenn dem nicht so wäre, würde es keine Menschheit geben.

\section{Die Grundpfeiler der Gesundheit}

Grundpfeiler der Gesundheit sind, dass Wunden heilen, gebrochene Knochen zusammenwachsen, zerstörtes Gewebe regeneriert, Abwehrkräfte sich im Immunsystem entwickeln und dieses zu einem guten Bekämpfer von Erkrankungen machen und dass alle Menschen mehrheitlich mit ausgewogener Psyche ausgerüstet sind. Zweifelsohne hat die Gesundheit beim Menschen Situationen durchzustehen, in denen sie zusätzlich unsere unterstützende Hilfe braucht. Damit werden aber vor allem die erwähnten Heilungsmöglichkeiten in Schwung gebracht und schadende Prozesse verzögert oder eliminiert. Die Folgen von Unfällen, die tagtäglich geschehen, werden überwunden, Verletzungen, die auf den Kriegsschauplätzen in aller Welt entstehen, werden, wenn immer möglich, kuriert, Wunden von operativen Eingriffen heilen, erlahmende Immunsysteme gegen Infektionen und Krebs werden erkannt und gestärkt, Behandlungen gegen neue Krankheiten und unerwünschte genetische Veränderungen werden gesucht und verwertet, und schliesslich stabilisieren sich degenerative Pro- zesse und psychische Auffälligkeiten unter kluger Anleitung.

Warum ist nun diese für die ärztliche Berufsethik so wichtige Krankheit durch die Gesundheit verdrängt worden, das Krankenhaus eben ein Gesundheitszentrum, das Krankengesetz ein Gesundheitsgesetz, die Krankenschwester zu einer Fachperson für Gesundheit geworden? Eine Erklärung dafür ist, dass Gesundheit ganz anders «besetzt ist» als Krankheit. Hinter dem

Durch die Jahre der Entwicklung von Leben auf dieser Erde hat sich auch unsere Gesundheit, also die Möglichkeit, gesund zu sein und zu bleiben, gebildet.

Begriff «Gesundheit» steht: Freiheiten haben, geniessen, Geld verdienen und sorglos leben können. Mit dem Begriff «Krankheit» verbinden wir hingegen Schicksalsschläge, Mühsal, Schmerzen, Einschränkungen jeglicher Art und Kosten. Der Bedeutungswechsel dieser gegensätzlichen Begriffe, so meine ich, ist damit nicht nur ein sprachliches, sondern auch ein zivilisatorisches Phänomen. Er geht einher mit der Ökonomisierung der heutigen Gesellschaft. Auch die Gesundheit wurde für den Markt entdeckt, zum käuflichen Gut gemacht. Sie passt so besser in unsere Gesellschaft, und ihre Kommerzialisierung findet fast keine Grenzen.

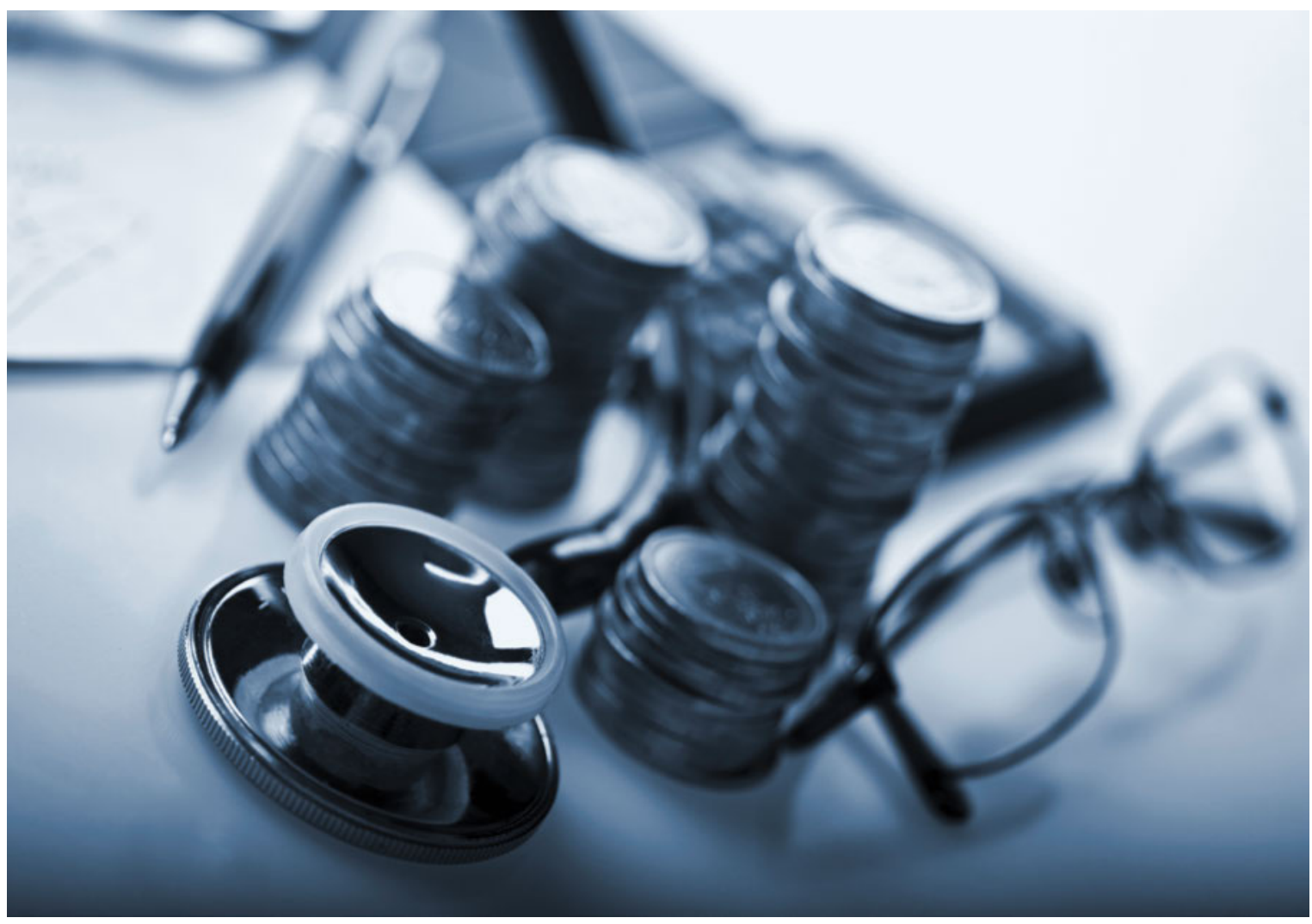

Die Gesundheit wird immer mehr zum käuflichen Gut. 
Ihre «Marktmenge» ist nicht limitiert. Dies ganz im Gegensatz zur Krankheit, die offensichtlich kein käufliches Gut sein kann und darum nicht marktfähig ist.

\section{Die Verbannung der Krankheit}

Das Gesundheitswesen ist ein wesentlicher Wirtschaftsfaktor geworden. Hinter ihm versteckt sich ein beängstigendes Wachstumspotential. In den USA zum Beispiel beträgt der Kostenanteil des Gesundheitswesens vom Bruttoinlandprodukt ca. $17 \%$, bei uns vorerst noch einen Drittel weniger, etwa $11,5 \%$. Reizen wir unser Gesundheitssystem mit den heute üblichen ökonomischen Mechanismen noch weiter aus, erreichen auch wir amerikanische Verhältnisse unter entsprechender Zunahme der Kosten. Der Gesundheitsmarkt fördert mit seiner Gewinnmaximierung auf allen Stufen diese Entwicklung. Ein Krankenmarkt würde dies nicht tun. In diesem würde ein Kranker mit individuellen Entscheiden medizinisch betreut, die vom ärztlichen Standpunkt aus gesehen Unnötiges von ihm fernhalten. Der Gesundheitsmarkt fordert dies nicht.

Diese Verbannung der Krankheit hat schliesslich mitgewirkt, dass nicht ökonomische Werte - in erster Linie Empathie, Gefühl für das Schicksalhafte und Solidarität -, die zum Verständnis jeglicher Erkrankung und gutem Umgang mit den Patienten wie auch mit den Ressourcen beitragen, in den Hintergrund traten. Der Gesundheitsmarkt stellte diesen Werten einzig die schwammige Forderung der Selbstverantwortung gegenüber. Aber: Wie sollen wir mit diesem Hinweis auf mehr Selbstverantwortung Eltern erklä-

\section{Das Gesundheitswesen ist ein wesentlicher Wirtschaftsfaktor geworden. Hinter ihm versteckt sich ein beängstigendes Wachstums- potential.}

ren, warum ihr Kind an einer Leukämie erkrankt, ein anderes bereits bei der Geburt einen Krebs im Auge hat? Ist der Velofahrer, der mit Helm, guter Fahrradbeleuchtung und korrekter Fahrweise in der regnerischen Nacht von einem Lastwagen angefahren und verletzt wird, unverantwortlich? Und müssen wir den sportbegeisterten Junior einer Fussballmannschaft schelten, wenn er ausgleitet und sich eine Verletzung zuzieht oder gar von seinem Trainer mit einer Infektionskrankheit angesteckt wird? Oder müssen wir einen Familienvater im mittleren Alter, der immer gesund lebte, sich vernünftig ernährte, nie rauchte, sich sportlich betätigte und auch beruflich keinem Stress

\section{Brauchen wir eine neue Standes- ordnung?}

Am 1. Juli 1997 wurde die Standesordnung FMH für verbindlich erklärt. Diese ersetzte die damals existierenden Standesordnungen der kantonalen Ärztegesellschaften. Die Standesordnung FMH kann somit dieses Jahr auf ihr 20-jähriges Bestehen zurückblicken.

Als Vorsitzender der damaligen Arbeitsgruppe kann ich aus meiner heutigen Sicht nicht gut beurteilen, was sie der FMH an klärenden Regelungen gebracht hat. Ihre Wirkung zeigte sich vor allem in den Beurteilungen der Standeskommissionen der kantonalen Ärzte- und Fachgesellschaften. So steht mir nicht zu, eine Kritik dieser Standesordnung aus heutiger Sicht anzubringen. Trotzdem denke ich, dass eine solche durchwegs angebracht sein könnte. Ich staune zum Beispiel über die Vielzahl qualitätssichernder Institutionen und Gremien, die über ärztliches Tun wachen und heute ohne Bezug zur Standesordnung FMH sind. Der Artikel «Wir Gesundheitsverkäufer» entstand aus der Erinnerung an eine kurze Diskussion, die wir vor 20 Jahren in unserer Arbeitsgruppe führten, heute aber mit unserer Standesordnung nur noch marginal zu tun hat. Er eignet sich aber ihres 20 -jährigen Bestehens zu gedenken.

unterworfen war, aber trotzdem an einer Herzkrankheit zu leiden begonnen hat, erklären, dass er selbst an seiner Krankheit schuld sei? Und wer ist schliesslich verantwortlich für einen hebephrenen Schub, in dem vieles zerschlagen wird?

\section{Die Krankheit soll im Mittelpunkt stehen}

Ich bin mir im Klaren, dass das Rad nicht zurückgedreht werden kann. Auch weiss ich, dass wir Ärzte und Ärztinnen viel und gut präventiv arbeiten. Wir dürfen aber nicht vergessen, dass immer die Kranken mit ihrer Krankheit im Mittelpunkt unserer Bemühungen stehen müssen. Wir haben unser ganzes Tun darauf zu richten, den Kranken zu helfen und uns um die Krankheiten mit individuellen Ansätzen zu kümmern. Dem Gesundheitsmarkt sollten wir nicht zu sehr vertrauen. So gelingt uns vielleicht sogar, dass wir das Rad so weit bremsen können, dass es nicht in einem Abgrund zerschellt oder wir, wie es kürzlich in dieser, unserer Zeitschrift stand, das System nicht an die Wand fahren [1]. Bleiben wir Ärzte und Ärztinnen für kranke Menschen, vermeiden wir, Gesundheitsverkäufer zu sein, und lassen wir uns auch nicht zu Gesundheitsverkäufern machen.

\section{Literatur}

1 Scholer M. «Wenn wir so weitermachen, fahren wir das System an die Wand.» Schweiz Ärztezeitung. 2017;98:1516.

Bildnachweis

(c) Nikilitov | Dreamstime.com 